\title{
Unmatched Projector/Backprojector Pairs: Perturbation and Convergence Analysis
}

\section{Elfving, Tommy; Hansen, Per Christian}

\section{Published in:}

S I A M Journal on Scientific Computing

Link to article, DOI:

$10.1137 / 17 \mathrm{M} 1133828$

Publication date:

2018

Document Version

Peer reviewed version

Link back to DTU Orbit

Citation $(A P A)$ :

Elfving, T., \& Hansen, P. C. (2018). Unmatched Projector/Backprojector Pairs: Perturbation and Convergence Analysis. S I A M Journal on Scientific Computing, 4O(1), A573-A591. https://doi.org/10.1137/17M1133828

\section{General rights}

Copyright and moral rights for the publications made accessible in the public portal are retained by the authors and/or other copyright owners and it is a condition of accessing publications that users recognise and abide by the legal requirements associated with these rights.

- Users may download and print one copy of any publication from the public portal for the purpose of private study or research.

- You may not further distribute the material or use it for any profit-making activity or commercial gain

- You may freely distribute the URL identifying the publication in the public portal

If you believe that this document breaches copyright please contact us providing details, and we will remove access to the work immediately and investigate your claim. 


\title{
UNMATCHED PROJECTOR/BACKPROJECTOR PAIRS: PERTURBATION AND CONVERGENCE ANALYSIS*
}

\author{
TOMME ELFVING ${ }^{\dagger}$ AND PER CHRISTIAN HANSEN H $^{\ddagger}$
}

\begin{abstract}
In tomographic reconstruction problems it is not uncommon that there are errors in the implementation of the forward projector and/or the backprojector, and hence we encounter a so-called unmatched projektor/backprojector pair. Consequently the matrices that represent the two projectors are not each others' transpose. Surprisingly, the influence of such errors in algebraic iterative reconstruction methods has received little attention in the literature. The goal of this paper is to perform a rigorous first-order perturbation analysis of the minimization problems underlying the algebraic methods, in order to understand the role played by the non-match of the matrices. We also study the convergence properties of linear stationary iterations based on unmatched matrix pairs, leading to insight into the behavior of some important row- and column-oriented algebraic iterative methods. We conclude with numerical examples that illustrate the perturbation and convergence
\end{abstract} results.

Key words. perturbation theory, convergence analysis, algebraic iterative reconstruction, semiconvergence, computed tomography

AMS subject classifications. $65 \mathrm{~F} 10,65 \mathrm{~F} 22$

1. Introduction. Among the many reconstruction methods in computed tomography (CT), algebraic iterative methods have received considerable interest due to their simplicity and their ability to adapt to the particular geometry of the CT scanner and the measurements. One of their applications is in limited-angle and limited-data CT, e.g., when exposition to a low dose of X-rays is an issue or when it is only possible to measure projection data for certain angles. These methods are therefore used for many reconstruction problems in imaging science [15], [17], [19].

Underlying the algebraic iterative methods is always a system of linear equations arising from the discretization of an ill-posed problem,

$$
A x=b, \quad A \in \mathbb{R}^{m \times n}, \quad b \in \mathbb{R}^{m} .
$$

This system is not necessarily consistent, and there are no restrictions on the rank or dimensions of $A$.

Well-known examples of algebraic iterative methods are Kaczmarz's method and variants of Landweber iteration [10], [14]. These methods, and their block extensions [24], utilize projection and backprojection operations in each iterative step. Both operations are defined by the geometry and the physics of the problem, and when discretized the projection is represented by the matrix $A$ in (1) while the backprojection is, in principle, represented by $A^{T}$ (the transpose of $A$ ).

However, the particular discretization methods used to obtain the projection and backprojection (see, e.g., [12], [16], [23], [26]) depend on the application and, to some extent, also on traditions in the specific application communities. The philosophy is that the discretized operations are approximations of the underlying physics, and

\footnotetext{
* Submitted to the editors DATE.

Funding: This work is a part of the project HD-Tomo funded by Advanced Grant No. 291405 from the European Research Council.

${ }^{\dagger}$ Department of Mathematics, Linköping University, SE-581 83 Linköping, Sweden (tommy.elfving@liu.se, https://liu.se/en/employee/tomel46/).

${ }^{\ddagger}$ Department of Applied Mathematics and Computer Science, Technical University of Denmark, DK-2800 Kgs. Lyngby, Denmark (pcha@dtu.dk, http://www.compute.dtu.dk/ pcha/).
} 
hence different discretization schemes may be appropriate for projection and backprojection.

Moreover, it is sometimes the case that the software uses different discretization methods for the projection and the backprojection, e.g., due to considerations about the most efficient use of multi-core processors, GPUs and other hardware accelerators. For example, this is the case in the software package ASTRA [27] when using GPU acceleration [20].

Consequently, in all these circumstances the matrix that represents the backprojection is not equal to $A^{T}$, a situation referred to as an unmatched projector/backprojector pair [25]. It is therefore relevant to study the influence of such an unmatched pair on the least squares and minimum-norm problems associated with (1), as well as their influence on the convergence properties of the algebraic iterative methods applied to the unmatched problem. Our analysis includes two important specific cases, namely, row- and column-iterations, including a semi-convergence analysis of these methods.

Our work is inspired by the work of Zeng and Gullberg [25] who also consider iterative reconstruction methods where the backprojection is replaced by a matrix that is very different from $A^{T}$ (such as a matrix that approximates filtered back projection). These scenarios are, however, outside the scope of our paper.

This paper is organized as follows. We first perform a first-order perturbation analysis of the minimization problems underlying the algebraic iterative methods, in order to understand the role played by the non-match of the matrices. We then study the convergence properties of linear stationary iterations based on unmatched matrix pairs, leading to insight into the behavior of some important row- and column-oriented algebraic iterative methods. We conclude with numerical examples that illustrate the perturbation and convergence results.

Throughout the paper we use the following notation: $I$ is an identity matrix of conforming size, $P_{\mathcal{S}}$ is the orthogonal projection matrix onto the subspace $\mathcal{S}, \mathcal{R}(A)$ and $\mathcal{N}(A)$ are the range and null space of $A$, respectively, $\rho(A)$ is the spectral radius of $A, \lambda_{j}(A)$ is an eigenvalue of $A$, and $\sigma_{r}(A)$ is the smallest nonsingular value of $A$.

2. First-Order Perturbation Analysis. We first perform a perturbation analysis of the minimization problems underlying the algebraic iterative methods. We consider the general case where both $A$ and $A^{T}$ are perturbed, reflecting situations where both matrices can be considered as discrete approximations of an underlying unknown exact operation.

2.1. The Unmatched Normal Equations. Recall the relation between a solution of the least squares problem and a solution of the normal equations [3]:

$$
x=\arg \min _{x}\|b-A x\|_{2} \quad \Leftrightarrow \quad A^{T} A x=A^{T} b .
$$

Let $\left\{A, A^{T}, \bar{b}\right\}$ be the unperturbed data, and put

$$
\tilde{A}=A+E_{1}, \quad \hat{A}^{T}=A^{T}+E_{2}, \quad b=\bar{b}+\delta b .
$$

Moreover, let $\bar{x}$ and $\bar{r}$ denote the unperturbed least squares solution and the corresponding residual, i.e.,

$$
A^{T} \bar{r}=0, \quad \bar{r}=\bar{b}-A \bar{x} .
$$

When we instead use the triple $\left\{\tilde{A}, \hat{A}^{T}, b\right\}$ we, in fact, aim at solving the equations

$$
\hat{A}^{T} \tilde{A}(\bar{x}+\delta x)=\hat{A}^{T} b \text {. }
$$


We will refer to (5) as the unmatched normal equations. Note that with $E_{2}=E_{1}^{T}$ we retrieve a classically perturbed least squares problem.

We remark that since $\mathcal{R}\left(Q^{T} Q\right)=\mathcal{R}\left(Q^{T}\right)$ for any matrix $Q$ the normal equations are always consistent. When $E_{2}=E_{1}^{T}$ then it follows that the symmetrically perturbed normal equations are also consistent. However, the unmatched normal equations (5) may not be consistent unless the perturbations are such that

$$
\hat{A}^{T} b \in \mathcal{R}\left(\hat{A}^{T} \tilde{A}\right) .
$$

If this does not hold we may choose to solve the unmatched equations in the least squares sense.

Using the notation in (3) we find

$$
\begin{aligned}
\hat{A}^{T} \tilde{A} & =A^{T} A+E, \quad \text { where } \quad E=A^{T} E_{1}+E_{2} A+E_{2} E_{1}, \\
\hat{A}^{T} b & =A^{T} \bar{b}+A^{T} \delta b+E_{2} b .
\end{aligned}
$$

Now using (4), (6) and (7) the unmatched normal equations (5) take the form

$$
\left(A^{T} A+E\right) \delta x=A^{T} \delta b+E_{2} b-E \bar{x} .
$$

To derive a first-order perturbation bound for $\delta x$ we need to use the pseudoinverse $A^{\dagger}$ which, as is well known, is not a continuous function of the elements of $A$ under rank-change. In order to ensure that $\delta x$ is a continuously differentiable function of the data we therefore impose the condition

$$
\operatorname{rank}\left(\hat{A}^{T} \tilde{A}\right)=\operatorname{rank}\left(A^{T} A+E\right)=\operatorname{rank}\left(A^{T} A\right) .
$$

Let us compare condition (9) with the corresponding condition for the least squares problem (where $\hat{A}^{T}=\tilde{A}^{T}$ ), cf. [3, section 1.4],

$$
\operatorname{rank}(\tilde{A})=\operatorname{rank}(A) .
$$

Since $\operatorname{rank}\left(\tilde{A}^{T} \tilde{A}\right)=\operatorname{rank}(\tilde{A})$ the condition (10) prevents rank-loss. However, for the unmatched problem we can only rely on the fact that

$$
\operatorname{rank}\left(\hat{A}^{T} \tilde{A}\right) \leq \min \left(\operatorname{rank}\left(\hat{A}^{T}\right), \operatorname{rank}(\tilde{A})\right) .
$$

Hence the rank conditions (9) are essential.

We now write (4) as

$$
g\left(\bar{x}, \bar{b}, A^{T}, A\right)=A^{T} \bar{b}-A^{T} A \bar{x}=0 .
$$

Since $g=0$ is constant (the constant being zero), its differential (sometimes also called the total derivative) w.r.t. $\bar{x}, \bar{b}, A^{T}$ and $A$ must be zero. It follows that

$$
E_{2}(A \bar{x})+A^{T}\left(E_{1} \bar{x}\right)+A^{T} A \delta x-E_{2} \bar{b}-A^{T} \delta b=0,
$$

or equivalently

$$
A^{T} A \delta x=A^{T}\left(\delta b-E_{1} \bar{x}\right)+E_{2} \bar{r} .
$$

An alternative way to derive this first-order error formula is to neglect higher-order error terms in (8). 
Note that when $E_{2} \bar{r} \in \mathcal{R}\left(A^{T}\right)$ this system is consistent. Otherwise we choose to solve (11) in the least squares sense. Using the relation $A^{\dagger}=\left(A^{T} A\right)^{\dagger} A^{T}$ we obtain from (11)

$$
\delta x=A^{\dagger}\left(\delta b-E_{1} \bar{x}\right)+\left(A^{T} A\right)^{\dagger} E_{2} \bar{r} .
$$

We remark that since

$$
A^{\dagger} \delta b=A^{\dagger} A A^{\dagger} \delta b=A^{\dagger} P_{\mathcal{R}(A)} \delta b
$$

only the component of $\delta b \in \mathcal{R}(A)$ contributes to the error (just as in the least squares case).

Let $\sigma_{1} \geq \sigma_{2} \geq \cdots \geq \sigma_{r}>0$ be the nonzero singular values of $A$, and without loss of generality we will assume that $\sigma_{1}=1$. We have

$$
\left\|A^{\dagger}\right\|_{2}=\frac{1}{\sigma_{r}}, \quad\left\|\left(A^{T} A\right)^{\dagger}\right\|_{2}=\frac{1}{\sigma_{r}^{2}} .
$$

We can summarize our results as follows.

Proposition 1. Assume that the rank conditions (9) hold. A first-order perturbation bound for the perturbation $\delta x$ of the solution to the unmatched normal equations (5) takes the form

$$
\|\delta x\|_{2} \leq \frac{1}{\sigma_{r}}\left(\left\|P_{\mathcal{R}(A)} \delta b\right\|_{2}+\left\|E_{1} \bar{x}\right\|_{2}\right)+\frac{1}{\sigma_{r}^{2}}\left\|E_{2} \bar{r}\right\|_{2} .
$$

If $\mathcal{R}\left(E_{2}\right) \subseteq \mathcal{R}\left(A^{T}\right)$ then the last term in (12) disappears.

If we compare the bound (12) with the corresponding bound for the least squares problem, the only difference is that $E_{1}^{T}$ is here replaced by $E_{2}$. As emphasized above, the rank conditions also differ in the two problems. We conclude that for inconsistent problems $(\bar{r}=\bar{b}-A \bar{x} \neq 0)$ it is specially important to keep the error in the backprojection small if one wants to stay close to the least squares solution.

2.2. The Unmatched Minimum-Norm Problem. The following relation between a solution of the dual least squares problem and a solution of the normal equations of the second kind is well known [3]:

$$
x=\arg \left\{\min _{x}\|x\|_{2} \mid A x=b\right\} \quad \Leftrightarrow \quad A A^{T} y=b, x=A^{T} y .
$$

Here it is assumed that the linear system (1) is consistent. Using the notation in (3), the perturbed dual problem becomes

$$
\tilde{A} \hat{A}^{T}(\bar{y}+\delta y)=\bar{b}+\delta b, \quad \bar{x}+\delta x=\hat{A}^{T}(\bar{y}+\delta y) .
$$

Here $\bar{y}$ and $\bar{x}$ denote the solutions corresponding to unperturbed data, i.e.,

$$
A A^{T} \bar{y}=\bar{b}, \quad \bar{x}=A^{T} \bar{y} .
$$

Let

$$
\tilde{A} \hat{A}^{T}=A A^{T}+F, \quad \text { where } \quad F=A E_{2}+E_{1} A^{T}+E_{1} E_{2} .
$$

Then, using (14) and (15), it follows that the perturbed second-kind normal equations take the form

$$
\left(A A^{T}+F\right) \delta y=\delta b-F \bar{y}, \quad \delta x=A^{T} \delta y+E_{2}(\bar{y}+\delta y) .
$$


Similarly as in (9) we impose the rank conditions

$$
\operatorname{rank}\left(\tilde{A} \hat{A}^{T}\right)=\operatorname{rank}\left(A A^{T}+F\right)=\operatorname{rank}\left(A A^{T}\right),
$$

which ensure that no rank change will occur in the perturbed problem. By differentiating $A A^{T} y=b$ we get

$$
A A^{T} \delta y=\delta b-E_{1}\left(A^{T} \bar{y}\right)-A\left(E_{2} \bar{y}\right) .
$$

If $\delta b-E_{1}\left(A^{T} \bar{y}\right) \in \mathcal{R}(A)$ this system is consistent. Otherwise we choose to solve (18) in the least squares sense, giving

$$
\delta y=\left(A A^{T}\right)^{\dagger} \delta b-\left(A A^{T}\right)^{\dagger} E_{1} A^{T} \bar{y}-\left(A A^{T}\right)^{\dagger} A E_{2} \bar{y} .
$$

Differentiating $x=A^{T} y$ gives $\delta x=E_{2} \bar{y}+A^{T} \delta y$. Inserting the expression for $\delta y$ and using $A^{\dagger}=A^{T}\left(A A^{T}\right)^{\dagger}$ it follows that

$$
\delta x=A^{\dagger} \delta b+\left(I-A^{\dagger} A\right) E_{2} \bar{y}-A^{\dagger} E_{1} A^{T} \bar{y} .
$$

Alternatively, this equation can be obtained by neglecting higher-order terms in (16). Thus we arrive at:

Proposition 2. Assume that the rank conditions (17) hold. A first-order perturbation bound for the perturbation $\delta x$ of the solution to the unmatched normal equations of the second kind (16) takes the form

$$
\|\delta x\|_{2} \leq \frac{1}{\sigma_{r}}\left(\|\delta b\|_{2}+\left\|E_{1} \bar{x}\right\|_{2}\right)+\left\|E_{2} \bar{y}\right\|_{2} .
$$

Hence we find that the unmatched minimum-norm solution is more sensitive to errors in $A$ than to errors in $A^{T}$ whereas, as shown above, the opposite is true for the unmatched least squares problem.

3. Convergence Analysis of Linear Stationary Iterations. Let $B \in \mathbb{R}^{n \times m}$ be a given matrix and put $C=B A$. We consider the following stationary iteration, with starting vector $x^{0}$, which we will refer to as the BA ITERATION,

$$
x^{k+1}=x^{k}+\mu B\left(b-A x^{k}\right)=T x^{k}+\mu B b:=F\left(x^{k}\right),
$$

with

$$
T=I-\mu C .
$$

Here $\mu>0$ is the relaxation parameter and $T$ is called the iteration matrix. Any fixed point $x^{*}$ of $F$ satisfies the equations

$$
C x^{*}=B b,
$$

where we will assume throughout the paper that $B b \in \mathcal{R}(C)$. We now characterize the limit point in a few cases.

- If $C$ is invertible then obviously $x^{*}=C^{-1} B b$.

- Next assume that $\mathcal{N}(C)=\mathcal{N}(A)$ and that $b \in \mathcal{R}(A)$. Then, with $b=A u$, it follows that $C\left(x^{*}-u\right)=0$ and hence $x^{*}-u \in \mathcal{N}(C)=\mathcal{N}(A)$ so that $A x^{*}=b$. 
- Another example is column iterations [8] where $B=M_{\mathrm{c}} A^{T}$ with $M_{\mathrm{c}}$ nonsingular. It follows that $A^{T} A x^{*}=A^{T} b$ so the fixed point is a least squares solution but not necessarily (unless $\mathcal{R}\left(M_{\mathrm{c}}\right) \subseteq \mathcal{R}\left(A^{T}\right)$ ) the one with minimal norm.

- Our final example is row iterations (see, e.g., the survey in [10]) where $B=A^{T} M_{\mathrm{r}}$ with $M_{\mathrm{r}}$ nonsingular. Then $A^{T} M_{\mathrm{r}} A x^{*}=A^{T} M_{\mathrm{r}} b$. Hence for inconsistent data the fixed point is not a (weighted) least squares solution, unless $M_{\mathrm{r}}$ is symmetric and positive definite.

Our goal here is to study convergence in the perspective of using non-matching matrices. A common situation is when there is noise in the right-hand side $b$. Let $\bar{x}^{k}$ be the iteration vector in (21) using the unperturbed right-hand side $\bar{b}$ and let $\bar{x}$ be a fixed point of the unperturbed iteration, i.e.,

$$
C \bar{x}=B \bar{b} .
$$

The total error can be decomposed into two terms

$$
x^{k}-\bar{x}=\left(x^{k}-\bar{x}^{k}\right)+\left(\bar{x}^{k}-\bar{x}\right) .
$$

The first term is called the noise error (or data error) and the second the iteration error. During the first iterations of a convergent method the iteration error dominates, and hence the total error decreases - but after a while the noise error starts to grow resulting in so-called semi-convergence [19].

We have already seen that the perturbation error in the final solution is proportional to the factor $\sigma_{r}^{-1}$. The noise error, on the other hand, measures the growth of the perturbation due to $\delta b$ during the iterations. The perturbation bound $\sigma_{r}^{-1}\|\delta b\|_{2}$ is problem dependent, and we will see that the noise error also depends on the choice of iteration method (i.e., the choice of $B$ ).

3.1. The Iteration Error. The following result from [22, Corollary 2.2] is adapted to our notation:

Proposition 3. The iterates $\left\{\bar{x}^{k}\right\}$ in the BA Iteration (21), using $b=\bar{b}$, converge to a solution of (24) if and only if $\rho(P T)<1$ with $P=P_{\mathcal{R}\left(C^{T}\right)}$.

Let $\lambda_{j}=\lambda_{j}(C)$ denote the $j$ th eigenvalue of $C=B A$. The matrix $C$ is, in general, not symmetric so that it may have complex eigenvalues, and since $A$ and $B$ are assumed real the complex eigenvalues of $C$ come in complex conjugate pairs. Let $i^{2}=-1$ and split the eigenvalues in real and imaginary parts, $\lambda_{j}=\Re\left(\lambda_{j}\right)+i \Im\left(\lambda_{j}\right)$.

Proposition 4. The iterates of the BA Iteration (21), using $b=\bar{b}$, converge to a solution of (24) if and only if

$$
0<\mu<\frac{2 \Re\left(\lambda_{j}\right)}{\left|\lambda_{j}\right|^{2}} \quad \text { and } \quad \Re\left(\lambda_{j}\right)>0
$$

Proof. Let $x=x_{\mathcal{N}}+x_{\mathcal{R}}$ with $x_{\mathcal{N}} \in \mathcal{N}(C)$ and $x_{\mathcal{R}} \in \mathcal{R}\left(C^{T}\right)$. First consider

$$
T x=x \quad \Leftrightarrow \quad C x=0 \quad \Leftrightarrow \quad x \in \mathcal{N}(C) .
$$

Hence the eigenvalue $\lambda=1$ is associated with the eigenspace $\mathcal{N}(C)$. Next consider $T x_{\mathcal{R}}=\lambda x_{\mathcal{R}}$. Then by Proposition 3 convergence occurs if and only if

$$
\left(1-\mu \Re\left(\lambda_{j}\right)\right)^{2}+\mu^{2} \Im\left(\lambda_{j}\right)^{2}<1,
$$

whence the result follows. 
Zeng and Gullberg [25] make a similar analysis for the case $\mathcal{N}(C)=\emptyset$ also implicitly assuming that $C$ has only real eigenvalues; their conclusion is therefore that the eigenvalues of $C$ should all be positive, and that $0<\mu<2 / \max \left(\lambda_{j}\right)$. If this is fulfilled they call the corresponding pair $(A, B)$ valid.

We now consider the iteration error $\bar{x}^{k}-\bar{x}$, and first assume that $N(C)=\emptyset$, so that the convergence criterion becomes $\rho(T)<1$. We have

$$
\bar{x}^{k}-\bar{x}=T^{k}\left(\bar{x}^{0}-\bar{x}\right),
$$

and it follows that

$$
\left\|\bar{x}^{k}-\bar{x}\right\|_{2} \leq\left\|T^{k}\right\|_{2}\left\|x^{0}-\bar{x}\right\|_{2} \leq\|T\|_{2}^{k}\left\|x^{0}-\bar{x}\right\|_{2} .
$$

In general we cannot assume that $\|T\|_{2}<1$ since $\rho(T) \leq\|T\|$ for any operator norm (for the 2-norm there holds equality if and only if $T$ is symmetric). Asymptotically, however, the convergence rate depends on the spectral radius due to the following classical result (for a proof see, e.g., [13, Theorem 2.1.1]):

Lemma 5. Assume that $\rho(T)<1$. Then for any operator norm

$$
\lim _{j \rightarrow \infty}\left\|T^{j}\right\|=\lim _{j \rightarrow \infty} \rho\left(T^{j}\right)=0 .
$$

In the case $\mathcal{N}(C) \neq \emptyset$ the iteration error $\bar{e}^{k}=\bar{x}^{k}-\bar{x}$ can be decomposed into two parts $\bar{e}_{\mathcal{N}}^{k} \in \mathcal{N}(C)$ and $\bar{e}_{\mathcal{R}}^{k} \in \mathcal{R}\left(C^{T}\right)$. Then $\bar{e}_{\mathcal{N}}^{k}$ (governed by the eigenvalue +1 ) remains unchanged through the iteration, whereas $\bar{e}_{\mathcal{R}}^{k}$ is governed by $\rho(P T)$ with $P$ from Proposition 3. So in both cases the convergence rate is linear. In [2, Theorem $2.15]$ it is shown that the asymptotic rate equals $\rho(P T)$ if and only if the corresponding eigenvalues are all semi-simple.

3.2. The Noise Error Due to $\delta b$. We next investigate how the errors $\delta b$ in the right hand side are propagated during the iterations. As mentioned previously the noise error is defined by

$$
e_{\mathrm{N}}^{k}=x^{k}-\bar{x}^{k},
$$

where $\bar{x}^{k}$ is the iteration vector using the unperturbed right-hand side $\bar{b}$.

By the iteration (21) we get $e_{\mathrm{N}}^{k+1}=T e_{\mathrm{N}}^{k}+\mu B \delta b$. Hence by induction, and assuming $e_{\mathrm{N}}^{0}=0$, it follows that

$$
e_{\mathrm{N}}^{k}=S_{k} \delta b \quad \text { with } \quad S_{k}=\mu \sum_{j=0}^{k-1} T^{j} B, \quad T=I-\mu B A .
$$

For later use we formulate (using that $\left(T^{j}\right)^{T}=\left(T^{T}\right)^{j}$ )

$$
\left\|S_{k}\right\|_{2}^{2}=\left\|S_{k} S_{k}^{T}\right\|_{2}=\mu^{2}\left\|\sum_{j=0}^{k-1} T^{j} B B^{T} \sum_{j=0}^{k-1}\left(T^{T}\right)^{j}\right\|_{2} .
$$

Now define the constant $c_{T}$ by

$$
\sup _{j}\left\|T^{j}\right\|_{2} \leq c_{T}
$$


The parameter $c_{T}$ is bounded when (26) holds. Further using (30), (32) it holds

$$
\left\|e_{\mathrm{N}}^{k}\right\|_{2} \leq \mu c_{T} k\|B \delta b\|_{2} \leq\left(\mu c_{T}\|B\|_{2}\right) k\|\delta b\|_{2} .
$$

It seems hard to derive sharper bounds for the general case. However for special choices of the matrix $B$ or special noise distributions the norm of the noise-error is bounded by a constant times $\sqrt{k}$. In Section 3.4 we consider three cases.

3.3. The Noise Error due to $E_{1}$ and $E_{2}$. We next study how the errors $E_{1}$ and $E_{2}$ propagate during the iterations. Let $\hat{B}=B+E_{2}, b=\bar{b}$ and $x^{k}=\bar{x}^{k}+\delta x^{k}$. We first consider the BA-iteration with ideal data:

$$
\bar{x}^{k+1}=\bar{x}^{k}-\mu B A \bar{x}^{k}+\mu B b .
$$

We assume (as previously) no rank-change in $\tilde{A}$ and $\hat{B}$. By computing the differential of $\bar{x}^{k+1}=\bar{x}^{k+1}\left(\bar{x}^{k}, B, A\right)$ w.r.t. $\bar{x}^{k}, B$ and $A$ we get

$$
\begin{aligned}
\delta x^{k+1} & =\delta x^{k}-\mu\left(E_{2} A \bar{x}^{k}+B E_{1} \bar{x}^{k}+B A \delta \bar{x}^{k}\right)+\mu E_{2} b \\
& =(I-\mu B A) \delta x^{k}+\mu E_{2}\left(b-A \bar{x}^{k}\right)-\mu B E_{1} \bar{x}^{k} .
\end{aligned}
$$

Alternatively, this equation can be derived by subtracting from Eq. (34) the corresponding iterations with perturbed data, and discarding higher-order terms (as in the previous section). Again let $T=I-\mu B A$, and put

$$
R=-\mu\left(E_{2} A+B E_{1}\right) .
$$

Then (35) becomes

$$
\delta x^{k+1}=T \delta x^{k}+R \bar{x}^{k}+\mu E_{2} b .
$$

Now put

$$
y^{k}=\left(\begin{array}{c}
\delta x^{k} \\
\bar{x}^{k}
\end{array}\right), \quad W=\left(\begin{array}{cc}
T & R \\
0 & T
\end{array}\right), \quad c=\left(\begin{array}{c}
c_{1} \\
c_{2}
\end{array}\right)=\mu\left(\begin{array}{c}
E_{2} b \\
B b
\end{array}\right) .
$$

Then (34) and (37) take the form

$$
y^{k+1}=W y^{k}+c .
$$

Assuming that $y^{0}=0$, i.e., $\delta x^{0}=x^{0}=0$, it follows that

$$
y^{k}=\sum_{j=0}^{k-1} W^{j} c
$$

It can be shown that

$$
W^{j}=\left(\begin{array}{cc}
T^{j} & R_{j} \\
0 & T^{j}
\end{array}\right), \quad R_{j}=\sum_{i=0}^{j-1} T^{j-i-1} R T^{i}
$$

and we note that $R_{j}$ is linear in $R$. From (40), (41) we obtain

$$
\delta x^{k}=\delta x_{1}^{k}+\delta x_{2}^{k}, \quad \delta x_{1}^{k}=\sum_{j=0}^{k-1} T^{j} c_{1}, \quad \delta x_{2}^{k}=\sum_{j=0}^{k-1} R_{j} c_{2} .
$$


For the first term we can write

$$
\delta x_{1}^{k}=\mu \sum_{j=0}^{k-1} T^{j} E_{2} b,
$$

and with the constant $c_{T}$ defined in (32) it follows that

$$
\left\|\delta x_{1}^{k}\right\|_{2} \leq\left(\mu c_{T}\|b\|_{2}\right) k\left\|E_{2}\right\|_{2} .
$$

We next consider the second term

$$
\delta x_{2}^{k}=\sum_{j=0}^{k-1} R_{j} c_{2}=\sum_{j=0}^{k-1} \sum_{i=0}^{j-1} T^{j-i-1} R T^{i} B b .
$$

It follows that

$$
\begin{aligned}
\left\|\delta x_{2}^{k}\right\|_{2} & \leq \sum_{j=0}^{k-1} \sum_{i=0}^{j-1}\left\|T^{j-i-1}\right\|_{2}\|R\|_{2}\left\|T^{i}\right\|_{2}\|B b\|_{2} \\
& \leq c_{T}^{2}\|B b\|_{2} \frac{k(k-1)}{2}\|R\|_{2} .
\end{aligned}
$$

We see that $\left\|\delta x_{2}^{k}\right\|_{2}$ is bounded by $k^{2}$ whereas $\left\|\delta x_{1}^{k}\right\|_{2}$ is bounded by $k$ (as is also the noise-error due to $\delta b$ as seen from (33)). We therefore consider the following estimation of $\delta x_{2}^{k}$. By induction we get from (37) (also assuming that $\delta x^{0}=0$ )

$$
\delta x^{k}=\sum_{j=0}^{k-1} T^{k-1-j}\left(R \bar{x}^{j}+\mu E_{2} b\right) .
$$

By taking norms we obtain

$$
\begin{aligned}
\left\|\delta x^{k}\right\|_{2} & \leq \sum_{j=0}^{k-1}\left\|T^{k-1-j}\right\|_{2}\left(\|R\|_{2}\left\|\bar{x}^{j}\right\|_{2}+\mu\left\|E_{2} b\right\|_{2}\right) \\
& \leq c_{T} \sum_{j=0}^{k-1}\left(\|R\|_{2}\left\|\bar{x}^{j}\right\|_{2}+\mu\left\|E_{2} b\right\|_{2}\right) \\
& \leq c_{T}\left(\|R\|_{2} \max _{j=0, \ldots, k-1}\left\|\bar{x}^{j}\right\|_{2}+\mu\left\|E_{2} b\right\|_{2}\right) k
\end{aligned}
$$

Since $\left\{\bar{x}^{k}\right\}$ is a convergent sequence $\left\|\bar{x}^{j}\right\|_{2}$ is bounded. Note that the first term is another bound for $\delta x_{2}^{k}$ whereas the second term corresponds to (44).

3.4. Special Cases. In this section we consider only perturbations of the righthand side $b$, and we focus on three special cases where we can derive sharper bounds for the noise error.

3.4.1. Case of a Special Right-Hand Side Perturbation.. Here we consider a general matrix $B$ but with a special perturbation of the right-hand side. Let

$$
\delta b=\delta b_{\mathcal{R}}+\delta b_{\mathcal{N}}, \quad \delta b_{\mathcal{R}} \in \mathcal{R}(A), \quad \delta b_{\mathcal{N}} \in \mathcal{N}\left(A^{T}\right),
$$

and note that there always exists a vector $\delta c$ such that we can write

$$
\delta b_{\mathcal{R}}=A \delta c .
$$


Below we will use the following matrix identity

$$
(I-X) \sum_{j=0}^{k-1} X^{j}=\sum_{j=0}^{k-1} X^{j}(I-X)=I-X^{k} .
$$

Proposition 6. Assume that $B \delta b_{\mathcal{N}}=0$ and that the iteration matrix $T$ is convergent. Then the noise error is bounded by

$$
\left\|e_{\mathrm{N}}^{k}\right\|_{2} \leq \sqrt{\mu c_{T}\left(1+c_{T}\right)\|B A\|_{2}} \sqrt{k}\|\delta c\|_{2} .
$$

Proof. We remark that the condition $B \delta b_{\mathcal{N}}=0$ guarantees that the perturbed system $B A x=B(\bar{b}+\delta b)$ is consistent. With $\delta b_{\mathcal{R}}=A \delta c$ and using assumption $B \delta b_{\mathcal{N}}=0$ it follows that $B \delta b=B \delta b_{\mathcal{R}}=B A \delta c$. Hence

$$
e_{\mathrm{N}}^{k}=\mu \sum_{j=0}^{k-1} T^{j} B A \delta c=\sum_{j=0}^{k-1} T^{j}(I-T) \delta c:=\widehat{S}_{k} \delta c .
$$

It follows

$$
\left\|\widehat{S}_{k}\right\|_{2}^{2}=\left\|\widehat{S}_{k}^{T} \widehat{S}_{k}\right\|_{2}=\left\|\left(\sum_{j=0}^{k-1} T^{j}(I-T)\right)^{T} \sum_{j=0}^{k-1} T^{j}(I-T)\right\|_{2}
$$

From (49) we obtain

$$
\left\|\widehat{S}_{k}\right\|_{2}^{2}=\left\|\left(\sum_{j=0}^{k-1} T^{j}(I-T)\right)^{T}\left(I-T^{k}\right)\right\|_{2}=\left\|\left(I-T^{T}\right) \sum_{j=0}^{k-1}\left(T^{T}\right)^{j}\left(I-T^{k}\right)\right\|_{2} .
$$

Using that $\left\|\left(T^{T}\right)^{j}\right\|_{2}=\left\|T^{j}\right\|_{2}$ and $\left\|I-T^{T}\right\|_{2}=\mu\left\|(B A)^{T}\right\|_{2}=\mu\|B A\|_{2}$ we get

$$
\left\|\hat{S}_{k}\right\|_{2}^{2} \leq \mu\|B A\|_{2} c_{T}\left(1+c_{T}\right) k,
$$

and hence the proof is complete.

REMARK 7. Note that the bound in (50) is expressed in $\delta c$ where $\delta c=A^{\dagger} \delta b_{\mathcal{R}}$. In cases where $\delta b_{\mathcal{R}}$ contains components corresponding to small singular values (typically high frequency components) this implies that $\|\delta c\|_{2}=O\left(1 / \sigma_{r}\right)\left\|\delta b_{\mathcal{R}}\right\|_{2}$.

Remark 8. Note that the assumption $B \delta b_{\mathcal{N}}=0$ in Proposition 6 is fulfilled for the special case (56) below. However, in general the bound (57) is more favorable than the bound (50) due to remark $\%$.

3.4.2. Block-Row Iterations.. Let $A$ be partitioned into $p$ disjoint block rows $R_{i}$, and let $b$ be partitioned accordingly. Further, let $\left\{\omega_{i}\right\}_{i=1}^{p}$ be a set of positive relaxation parameters and let $M_{i} \in \mathbb{R}^{m_{i} \times m_{i}}, i=1,2, \ldots, p$ be a set of given symmetric positive definite matrices. Consider the iteration

$$
\begin{aligned}
z^{0} & =x^{k}, \\
z^{i} & =z^{i-1}+\omega_{i} R_{i}^{T} M_{i}\left(b_{i}-R_{i} z^{i-1}\right), \quad i=1,2, \ldots, p, \\
x^{k+1} & =z^{p} .
\end{aligned}
$$


By different choices of $M_{i}$ many well-known block-row iterations appear. With $M_{i}=$ $\left(R_{i} R_{i}^{T}\right)^{-1}$ we get the Kaczmarz iteration [15], [19]. With $M_{i}=1 / m_{i}\left(\operatorname{diag}\left(R_{i}^{T} R_{i}\right)\right)^{-1}$, we get the Cimmino method (assuming equal weights). Note that the Cimmino method can be considered as using a diagonal approximation of the corresponding matrix in Kaczmarz's method. Other examples are BiCav [5], SART [1] and DROP [4] (for more details see, e.g., [10]). Let

$$
M_{\mathrm{r}}=\left(D_{\mathrm{r}}+L_{\mathrm{r}}\right)^{-1}, \quad \text { with } \quad D_{\mathrm{r}}=\operatorname{diag}\left(\omega_{i}^{-1} M_{i}^{-1}\right), \quad L_{\mathrm{r}}=\operatorname{slt}\left(A A^{T}\right),
$$

where $\operatorname{slt}(Q)$ denotes the strictly lower triangular part of $Q$. Then $[9$, Proposition 4]:

$$
x^{k+1}=x^{k}+A^{T} M_{\mathrm{r}}\left(b-A x^{k}\right) .
$$

Hence this is an instance of the BA Iteration (21) with $\mu=1$. It is known (see [6], [9]) that the method converges if

$$
\omega_{i} \in\left(\epsilon,(2-\epsilon) / \rho\left(R_{i}^{T} M_{i} R_{i}\right)\right), \quad i=1,2, \ldots, p, \quad 0<\epsilon<2 .
$$

Assuming (51) is satisfied, we may conclude from Proposition 4 (note that the convergence conditions there are both necessary and sufficient) that the spectrum of $A^{T} M_{\mathrm{r}} A$ is contained in the positive halfspace of the complex plane. A direct proof of this fact appears in [18, Lemma 3.1]. To allow for an (outer) relaxation parameter $\mu \neq 1$ we form $x^{k+1}=(1-\mu) x^{k}+\mu z^{p}$ which yields

$$
x^{k+1}=x^{k}+\mu A^{T} M_{r}\left(b-A x^{k}\right) .
$$

We stress, even in the case when $\omega_{i}=\omega$, that $\mu$ and $\omega$ are two independent relaxation parameters, since then $M_{\mathrm{r}}=\omega\left(\operatorname{diag}\left(M_{i}^{-1}\right)+\omega L_{\mathrm{r}}\right)^{-1}$. Hence one cannot just merge $\mu \omega$ into a single relaxation parameter since $\omega$ also affects $L_{\mathrm{r}}$.

Expressions for the noise error, assuming $\mu=1$, were recently presented in [7] and independently in [18]. We will next shortly discuss and compare these bounds. In [7] a bound of the form $c \sqrt{k}\|\delta b\|_{2}$ is derived (and also for variants of the algorithm that incorporate a projection on a convex set). However, the constant $c \sim 1 / \sigma_{r}\left(M_{\mathrm{r}} A\right)$ usually grossly overestimates the real noise error. Kindermann and Leitao [18, Lemma $3.2]$ also derived a bound of this form with a constant $c$ not depending on $1 / \sigma_{r}\left(M_{r} A\right)$; however they then need to assume that

$$
\sup _{j}\left\|Q^{j}\right\|_{2} \leq c_{Q}, \quad \text { where } \quad Q=I-M_{\mathrm{r}} A A^{T}
$$

with $c_{Q}$ bounded. Note that the convergence condition (for exact data) is

$$
\sup _{j}\left\|T_{\mathrm{r}}^{j}\right\|_{2} \leq c_{T_{\mathrm{r}}}, \quad \text { where } \quad T_{\mathrm{r}}=I-A^{T} M_{\mathrm{r}} A
$$

with $c_{T_{\mathrm{r}}}$ bounded. There is no simple relation between $\left\|Q^{j}\right\|_{2}$ and $\left\|T_{\mathrm{r}}^{j}\right\|_{2}$ and hence Lemma 5 does not imply that $c_{Q}$ is bounded. However, in [18] a sufficient condition is derived which assures that $c_{Q}$ is bounded. Before stating this result we need to resolve some notational differences between [18] and [8]-[10]. In [18] the equations are scaled and $\omega_{i}=\omega=1$ is assumed. Put $\bar{A}=D_{\mathrm{r}}^{-1 / 2} A$, and let $\bar{L}_{\mathrm{r}}=\operatorname{slt}\left(\bar{A} \bar{A}^{T}\right)$. Then, according to [18, Lemma 3.8], $c_{Q}$ is bounded if

$$
\left\|\bar{L}_{r}\right\|_{2}+1 / 2\left\|\bar{A} \bar{A}^{T}\right\|_{2}<1 \text {. }
$$

The introduction of $\mu$ in (53)-(54) does not affect condition (55) as seen by inspecting [18, Lemma 3.8]. 
3.4.3. Block-Column Iterations. Let $M_{\mathrm{c}} \in R^{n \times n}$ be a given, not necessarily symmetric, nonsingular matrix, and consider the choice

$$
B=M_{\mathrm{c}} A^{T} \quad \text { such that } T_{\mathrm{c}}=I-\mu M_{\mathrm{c}} A^{T} A .
$$

For $\mu=1$ this case includes a class of block-column sequential iterations recently studied in [8] (see also [3], [21]); among its members are SOR, column-Cimmino and column-BiCav.

Conditions (involving $M_{\mathrm{c}}$ ) guaranteeing convergence towards a least squares solution for exact data are given in [8]. Assuming these conditions we may also conclude from Proposition 4 that the spectrum of $M_{\mathrm{c}} A^{T} A$ is contained in the positive halfspace of the complex plane. It is quite straightforward to also introduce the outer relaxation parameter $\mu$ in the column iteration scheme. Then one defines the new iterate as a convex combination of the old and new iterates. One also needs to generate the corresponding residual in the same fashion.

We next derive a bound for the noise error. From (31) and (56) we get

$$
\left\|S_{k}\right\|_{2}^{2}=\left\|\sum_{j=0}^{k-1} T_{\mathrm{c}}^{j} \mu M_{\mathrm{c}}\left(A^{T} A \mu M_{\mathrm{c}}^{T}\right) \sum_{j=0}^{k-1}\left(I-A^{T} A \mu M_{\mathrm{c}}^{T}\right)^{j}\right\|_{2} .
$$

It follows by (49) with $X=I-A^{T} A \mu M_{\mathrm{c}}^{T}$ that

$$
\left(A^{T} A \mu M_{\mathrm{c}}^{T}\right) \sum_{j=0}^{k-1}\left(I-A^{T} A \mu M_{\mathrm{c}}^{T}\right)^{j}=I-\left(I-A^{T} A \mu M_{\mathrm{c}}^{T}\right)^{k}=I-\left(T_{\mathrm{c}}^{T}\right)^{k} .
$$

Hence (noting that $\left\|T_{\mathrm{c}}^{j}\right\|_{2}=\left\|\left(T_{\mathrm{c}}^{T}\right)^{j}\right\|_{2}$ )

$$
\left\|S_{k}\right\|_{2}^{2}=\left\|\sum_{j=0}^{k-1} T_{\mathrm{c}}^{j} \mu M_{\mathrm{c}}\left(I-\left(T^{T}\right)^{k}\right)\right\|_{2} \leq c_{T_{\mathrm{c}}} \mu\left\|M_{\mathrm{c}}\right\|_{2}\left(1+c_{T_{\mathrm{c}}}\right) k .
$$

This leads to the following result.

Proposition 9. Assume that $B=\mu M_{\mathrm{c}} A^{T}$ and that the corresponding iteration matrix $T_{\mathrm{c}}$ is convergent. Then the noise error is bounded by

$$
\left\|e_{\mathrm{N}}^{k}\right\|_{2} \leq \sqrt{\mu c_{T_{\mathrm{c}}}\left(1+c_{T_{\mathrm{c}}}\right)\left\|M_{\mathrm{c}}\right\|_{2}} \sqrt{k}\|\delta b\|_{2} .
$$

Note that, in contrast to the row iteration, the iteration error and the noise error are governed by the same quantity $\left\|T_{\mathrm{c}}^{j}\right\|_{2}$.

We finally remark that for the special case $M_{\mathrm{c}}=I$ (Landweber iteration) we retrieve the result by Engl, Hanke and Neubauer [11, Lemma 6.2]. Instead of the factor $\sqrt{c_{T_{\mathrm{c}}}\left(1+c_{T_{\mathrm{c}}}\right)}$ they get, based on their assumptions, the factor one.

4. Numerical Examples. We conclude with numerical examples that illustrate some of the points made in this work. We first consider the general perturbation bounds in Propositions 1-2, and then we turn to the behavior of the BA ITERATION (21) under perturbations. In all our experiments, the matrix $A$ was generated by means of the function paralleltomo from AIR Tools [14]; it is a sparse matrix that represents a discretization of the Radon transform, and we scaled the matrix such that the largest singular value equals 1 . Moreover, we generated the exact data as $\bar{b}=A \bar{x}$, where $\bar{x}$ represents the Shepp-Logan phantom generated by MATLAB's phantom function. The image is $64 \times 64$, leading to an exact solution $\bar{x} \in \mathbb{R}^{4096}$ with $\|\bar{x}\|_{2}=15.8$. 
TABLE 1

The different combinations of perturbations of $\bar{b}, A$ and $A^{T}$ that contribute to the perturbation bound (12) for the least squares problem.

\begin{tabular}{|c|ccccccc|ccccccc|} 
Case & 1 & 2 & 3 & 4 & 5 & 6 & 7 & 8 & 9 & 10 & 11 & 12 & 13 & 14 \\
\hline$\delta b$ & $\mathrm{X}$ & 0 & $\mathrm{X}$ & 0 & $\mathrm{X}$ & 0 & $\mathrm{X}$ & $\mathrm{X}$ & 0 & $\mathrm{X}$ & 0 & $\mathrm{X}$ & 0 & $\mathrm{X}$ \\
$E_{1}$ & 0 & $\mathrm{X}$ & $\mathrm{X}$ & 0 & 0 & $\mathrm{X}$ & $\mathrm{X}$ & 0 & $\mathrm{X}$ & $\mathrm{X}$ & 0 & 0 & $\mathrm{X}$ & $\mathrm{X}$ \\
$E_{2}$ & 0 & 0 & 0 & $\mathrm{X}$ & $\mathrm{X}$ & $\mathrm{X}$ & $\mathrm{X}$ & 0 & 0 & 0 & $\mathrm{X}$ & $\mathrm{X}$ & $\mathrm{X}$ & $\mathrm{X}$ \\
$\bar{r}$ & 0 & 0 & 0 & 0 & 0 & 0 & 0 & $\mathrm{X}$ & $\mathrm{X}$ & $\mathrm{X}$ & $\mathrm{X}$ & $\mathrm{X}$ & $\mathrm{X}$ & $\mathrm{X}$ \\
\hline
\end{tabular}
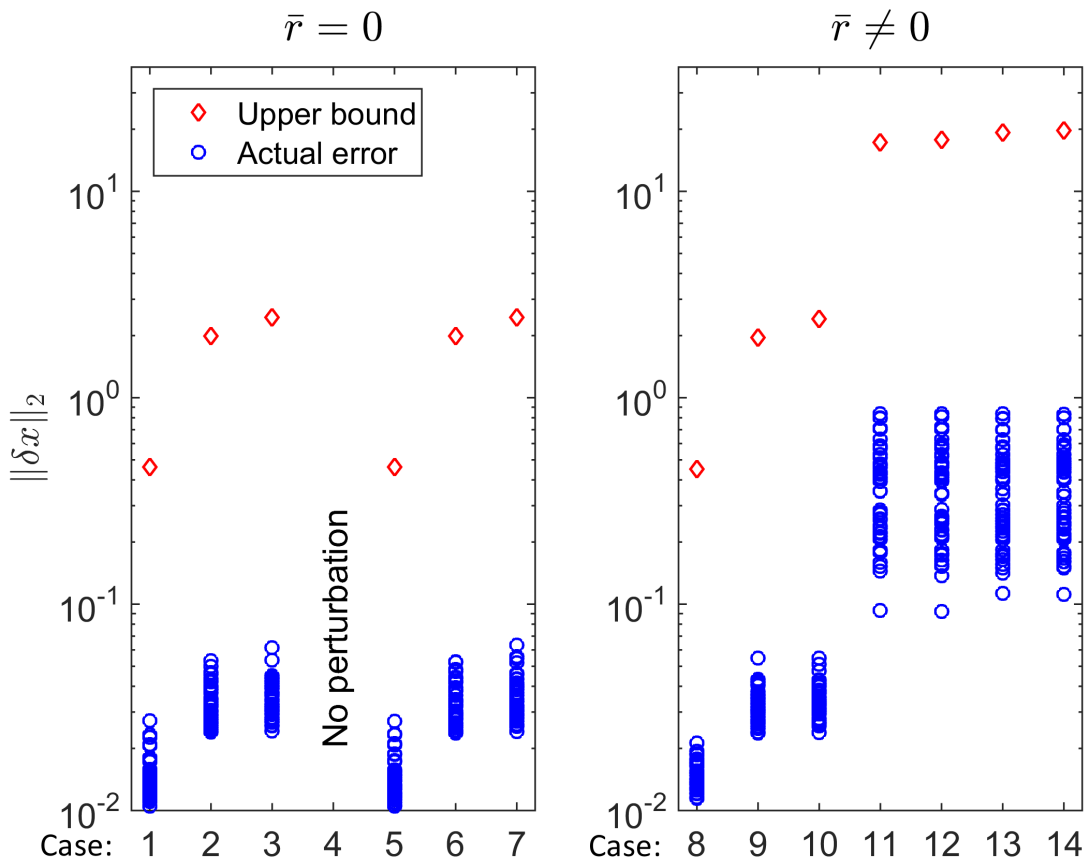

FIG. 1. The actual errors and the upper bounds (12) for the least squares problem, for 50 random perturbations of an overdetermined full-rank problem; see Table 1 for details about the 14 cases.

4.1. Sensitivity. All perturbed solutions to the unmatched normal equations (5), as well as the dual problem for the unmatched normal equations of the second kind (16), were computed by means of MATLAB's "backslash." These solutions were used to compute the actual errors shown in Figures 1 and 2 below. All the involved matrices have full rank, and in particular the rank conditions (9) and (17) are satisfied.

We first study overdetermined systems, for which the perturbation bound is given by (12). The test problem here uses 180 projection angles $1^{\circ}, 2^{\circ}, \ldots, 180^{\circ}$ and 91 detector pixels, giving a matrix of dimensions $m \times n=16,380 \times 4,096$. The smallest singular value of $A$ is $\sigma_{r}=9.90 \cdot 10^{-4}$.

To study how well the upper bound describes the actual error, we generated 50 instances of perturbed problems with Gaussian perturbations scaled such that:

$$
\|\delta b\|_{2} /\|\bar{b}\|_{2}=10^{-4}, \quad\left\|E_{1}\right\|_{\mathrm{F}} /\|A\|_{\mathrm{F}}=\left\|E_{2}\right\|_{\mathrm{F}} /\|A\|_{\mathrm{F}}=10^{-3} .
$$

We considered both consistent problems (with $\bar{r}=0$ ) and inconsistent systems with $\bar{r} \perp \bar{b}$ and $\|\bar{r}\|_{2} /\|\bar{b}\|_{2}=0.03$. The different combinations of perturbations of $\bar{b}, A$ 
TABLE 2

The different combinations of perturbations of $\bar{b}, A$ and $A^{T}$ that contribute to the perturbation bound (20) for the minimum-norm problem - same cases as in Table 1.

\begin{tabular}{|c|ccccccc|} 
Case & 1 & 2 & 3 & 4 & 5 & 6 & 7 \\
\hline$\delta b$ & $\mathrm{X}$ & 0 & $\mathrm{X}$ & 0 & $\mathrm{X}$ & 0 & $\mathrm{X}$ \\
$E_{1}$ & 0 & $\mathrm{X}$ & $\mathrm{X}$ & 0 & 0 & $\mathrm{X}$ & $\mathrm{X}$ \\
$E_{2}$ & 0 & 0 & 0 & $\mathrm{X}$ & $\mathrm{X}$ & $\mathrm{X}$ & $\mathrm{X}$ \\
\hline
\end{tabular}

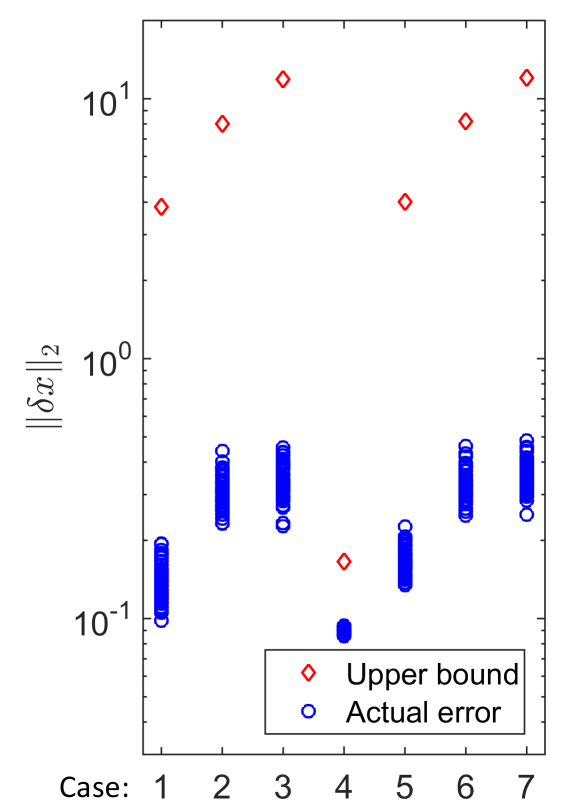

FIG. 2. The actual errors and the upper bounds (20) for the minimum-norm problem, for 50 random perturbations of an underdetermined full-rank problem; see Table 2 for details about the 7 cases.

and $A^{T}$ are listed in Table 1 and the results are shown in Figure 1. (Case 4 with $\delta b=0, E_{1}=0, E_{2} \neq 0$ and $\bar{r}=0$ gives the exact solution $\bar{x}$ except for rounding errors.) Our results confirm that the upper bounds track the actual errors (but are quite pessimistic) and that the errors are indeed larger for inconsistent systems in the presence of perturbations of $A^{T}$.

Next, we study minimum-norm solutions to underdetermined problems, whose perturbation bound is given by (20). The test problem here uses 45 projection angles $4^{\circ}, 8^{\circ}, \ldots, 180^{\circ}$ and 91 detector pixels, giving a matrix of dimensions $m \times n=2,745 \times$ 4,096. Both $A$ and $B$ have full rank, and the smallest singular value of $A$ is $\sigma_{r}=$ $4.37 \cdot 10^{-3}$

Again, we generated 50 instances of perturbed problems with Gaussian perturbations scaled as above, and with $\|\bar{y}\|_{2}=1145$. The different combinations of perturbations of $\bar{b}, A$ and $A^{T}$ are listed in Table 2 and the results are shown in Figure 2. Similar to before, the upper bounds track the actual errors (but are quite pessimistic) and our results confirm that he errors are indeed smaller for problems where the errors are confined to $A^{T}$. 


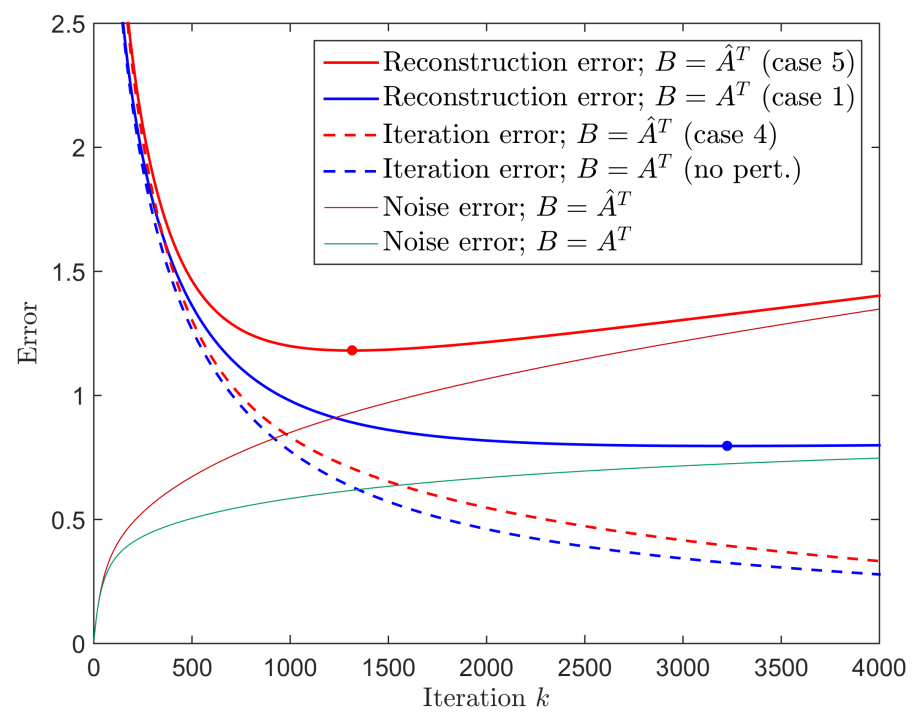

Fig. 3. We show three types of errors for the BA ITERATION (21) with both matched and unmatched transpose. The thick solid lines show the reconstruction errors $\left\|x^{k}-\bar{x}\right\|_{2}$ for the test problem with noise in the data $b$, and the minima are marked with the bullets. The thick dashed lines show the iteration errors $\left\|\bar{x}^{k}-\bar{x}\right\|_{2}$, i.e., the reconstruction errors without noise in the data. The thin solid lines show the noise errors $\left\|e_{\mathrm{N}}^{k}\right\|_{2}$. It is evident that there is semi-convergence, because the total reconstruction error is the sum of the iteration error and the noise error.

4.2. Convergence and Semi-Convergence. We now focus on the behavior of the BA ItERATion (21) in Section 3 with a unmatched transpose, using the $16,380 \times 4,096$ test problem from before with $\bar{r}=0$. The unmatched transpose $\hat{A}^{T}$ was generated from $A^{T}$ by neglecting small elements, such that the number of non-zeros in $\hat{A}$ is approximately half of that in $A$ and $\left\|E_{2}\right\|_{\mathrm{F}} /\|A\|_{\mathrm{F}}=\|A-\hat{A}\|_{\mathrm{F}} /\|A\|_{\mathrm{F}}=0.406$. Noisy data $b=\bar{b}+\delta b$ was generated by adding Gaussian white noise $\delta b$ scaled such that $\|\delta b\|_{2} /\|\bar{b}\|_{2}=0.01$. In all our numerical tests - for both triples $\left\{A, A^{T}, \bar{b}\right\}$ and $\left\{A, \hat{A}^{T}, b\right\}$ - we used $\mu=1.9 /\left\|A^{T} A\right\|_{2}=1.9$ (due to our scaling of $A$ ).

Both $A$ and $\hat{A}$ have full rank, and all real parts of the eigenvalues of $C=\hat{A}^{T} A$ are positive (the smallest real part is $9.35 \cdot 10^{-7}$ ). For the unperturbed right-hand side $\bar{b}=A \bar{x}$, the BA Iteration (21) with both $B=A^{T}$ and $B=\hat{A}^{T}$ converges to $\bar{x}$ (because $C=B A$ has full rank and $C^{-1} B \bar{b}=(B A)^{-1} B A \bar{x}=\bar{x}$ ). For the perturbed right-hand side, iteration (21) converges to the least squares solution $\bar{x}$ when $B=A^{T}$ and to a solution of (5) when $B=\hat{A}^{T}$.

Figure 3 shows results for the BA ITERATION (21) with both matched transpose $B=A^{T}$ and unmatched transpose $B=\hat{A}^{T} \neq A^{T}$ :

- The thick solid lines are the reconstruction errors $\left\|x^{k}-\bar{x}\right\|_{2}$, where $\bar{x}$ denotes the exact phantom image.

- The thick dashed lines are the iteration errors $\left\|\bar{x}^{k}-\bar{x}\right\|_{2}$, i.e., the reconstruction errors without noise in the data.

- The thin solid lines are the noise errors $\left\|e_{\mathrm{N}}^{k}\right\|_{2}$

In the case of noise-free data we see that both iterations converge, and the iteration with the unmatched transpose converges slower. When noise is present in the data, the iteration with the unmatched transpose reaches the point of semi-convergence after 1314 iterations where the minimum reconstruction error is 1.181. This error is $48 \%$ 


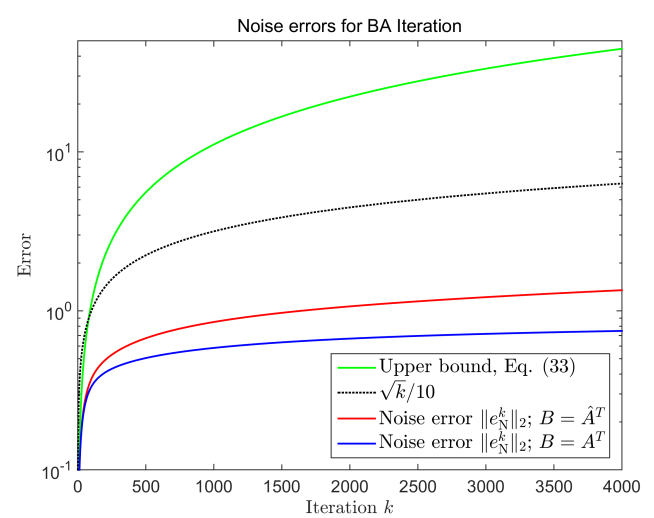

FIG. 4. The norm of the noise error $\left\|e_{\mathrm{N}}^{k}\right\|_{2}$ for the BA ITERATION (21), and the corresponding upper bound in (33). It appears that $\left\|e_{\mathrm{N}}^{k}\right\|_{2}$ is more likely to be proportional to $\sqrt{k}$.
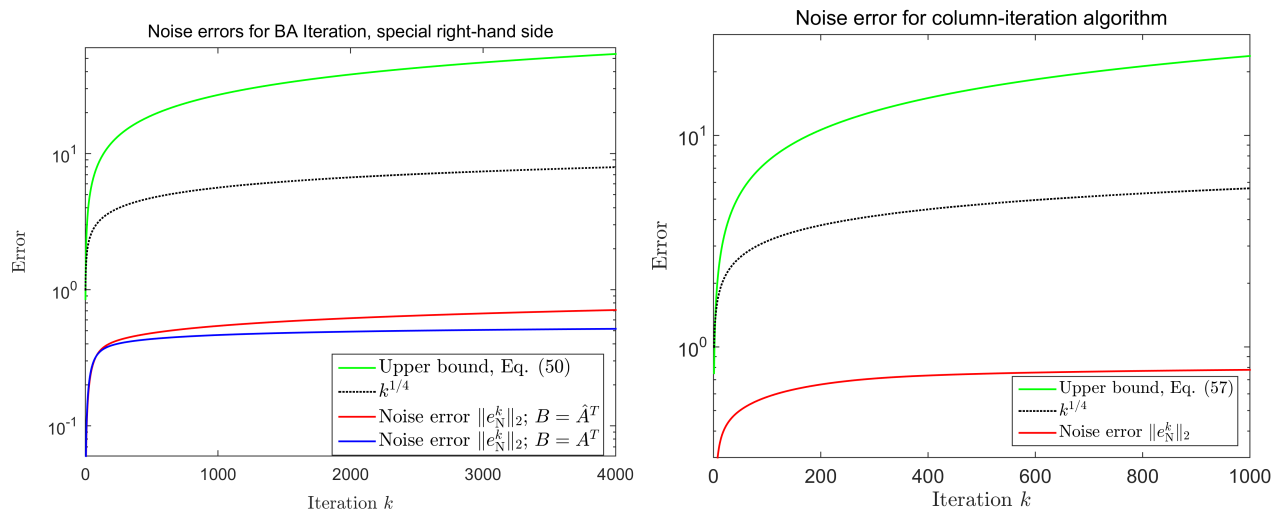

FIG. 5. The norm of the noise error $\left\|e_{\mathrm{N}}^{k}\right\|_{2}$ for the BA ITERATION (21), and the corresponding upper bound, for two special cases. Left: the special right-hand side perturbation considered in $\S 3.4 .1$ where the upper bound is given by (50). Right: the column-iteration algorithm from $\$ 3.4 .3$ whose upper bound is given by (57). Both upper bounds are proportional to $\sqrt{k}$, but it appears that the noise-error norms increase slower than that.

larger than the minimum error 0.796 for the iterations with the matched transpose, achieved after 3225 iterations.

This example clearly illustrates two important issues related to the use of an unmatched transpose: the convergence can be slower, and for noisy data the smallest achievable error (at the point of semi-convergence) can be larger than when using the matched transpose.

Next we show numerical examples related to the results in Section 3.2 about the noise error due to perturbations of the right-hand side; we use the same test problem as above. The results in Figure 4 (note the semi-logarithmic axis) supplement the results in Figure 3. Here we compare the norm of the noise error $\left\|e_{\mathrm{N}}^{k}\right\|_{2}$ for the BA ITERATION (21), with both the matched and unmatched transpose, with the rather pessimistic upper bound in Eq. (33) which is proportional to $k$ (in this example $\left.c_{T}=1.15\right)$. For reference we also show a plot of $\sqrt{k} / 10$ indicating that $\left\|e_{\mathrm{N}}^{k}\right\|_{2}$ is more likely to be proportional to $\sqrt{k}$. Note that when $B=A^{T}$ (the Landweber case) then the noise error indeed behaves like $O(\sqrt{k})$, as remarked at the end of $\S 3.4 .3$. 


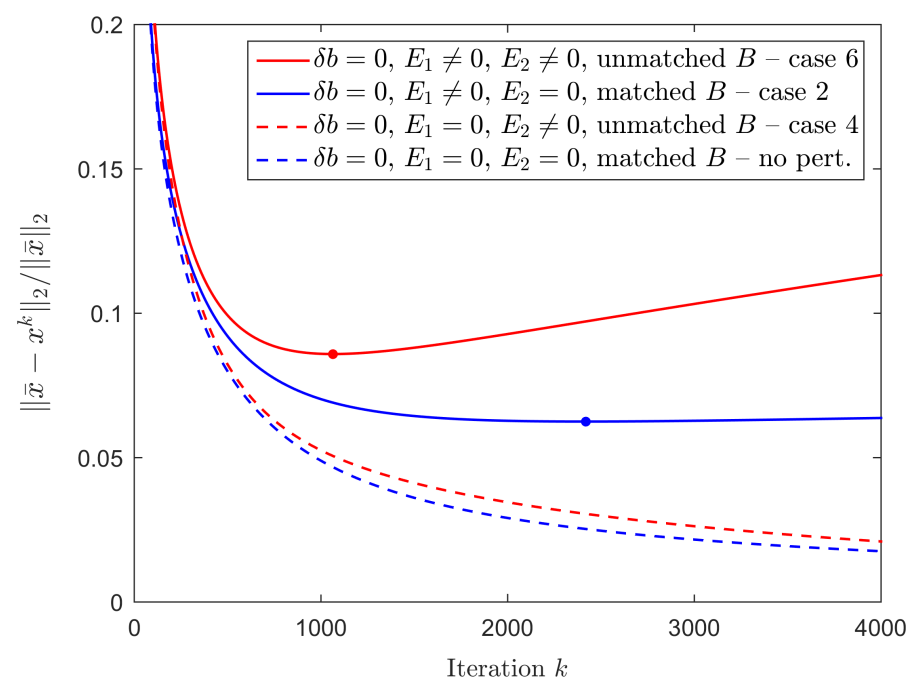

FIG. 6. Reconstruction errors for the BA ITERATION (21) for an example with errors in the matrices $A$ and $B$ (but not in the right-hand side); cases 2, 4 and 6 refer to Table 1. Matrix errors also lead to semi-convergence, and the minimum reconstruction error is larger for the unmatched transpose.

In Figure 5 we show results for two special cases, namely, the special right-hand side considered in $\S 3.4 .1$, and the column-iteration method (here with block size one) considered in §3.4.3. In the former case we scaled the perturbations such that $\|\delta c\|_{2}=$ 0.370 and $\left\|\delta b_{\mathcal{R}}\right\|_{2} /\|\bar{b}\|_{2}=\left\|\delta b_{\mathcal{N}}\right\|_{2} /\|\bar{b}\|_{2}=0.005$, and we have $c_{T}=1.15$. In the latter case we have $\left\|M_{\mathrm{c}}\right\|_{2}=1.77 \cdot 10^{-3}$ and $c_{T_{\mathrm{c}}}=1.43$. In both examples the upper bounds are proportional $\sqrt{k}$, but it appears that the noise-error norms grow slower - perhaps like $k^{1 / 4}$.

We conclude with a numerical example that illustrates the influence of matrix errors on the semi-convergence, cf. Section 3.3, using the same $A, B$ and $b$ as before. There are no errors in the right-hand side in this example $(\delta b=0)$. The perturbation $E_{1}$ of $A$ has the same sparsity pattern as $A$, the nonzero elements of $E_{1}$ have a Gaussian distribution, and $E_{1}$ is scaled such that $\left\|E_{1}\right\|_{\mathrm{F}} /\|A\|_{\mathrm{F}}=0.05$. The perturbed matrix $B+E_{2}$ is generated by introducing zeros in $\left(A+E_{1}\right)^{T}$ in the same positions as those introduced in $A^{T}$ to produce $B$; then $\left\|E_{2}\right\|_{\mathrm{F}} /\|B\|_{\mathrm{F}}=0.041$. Figure 6 shows the error histories for the BA Iteration (21) for cases 2, 4 and 6 from Table 1 as well as with no errors. We see that matrix perturbations - for both the matched and the unmatched $B$ - have the same effect as perturbations of the right-hand side, namely, they lead to semi-convergence. Moreover, with an unmatched transpose the minimum reconstruction error is larger than with a matched transpose.

5. Conclusion. We studied the influence of errors in the two matrices $A$ and $B$ that represent the forward projector and the backprojector, respectively, in computed tomography. This includes the important case where an algebraic iterative method is implemented such that the computed backprojection $B$ is not identical to a multiplication with $A^{T}$, where $A$ is the forward projection.

We first performed a first-order perturbation analysis of the unmatched normal equations associated with the perturbed matrices; this analysis augments the classical analysis of least squares problems. Our analysis shows that the errors in the two matri- 
ces have different effects in the minimization problems underlying the reconstructions: the least squares solution is more sensitive to errors in $B$ than in $A$ (Proposition 1), while the opposite is true for the minimum-norm solution (Proposition 2).

We also considered linear stationary iterations based on unmatched matrix pairs. For certain choices of $B$, these iterations are equivalent to known methods such as Kaczmarz, column-iteration, Cimmino and SIRT as well as their block versions. We derived bounds for the errors in the iteration vectors, for both the generic case and for some important special cases. In particular we show that the upper bound for the noise error increases with $k$ in the generic case, and with $\sqrt{k}$ for block-column iterations and for a special right-hand side perturbation.

Finally, we presented numerical examples which demonstrate that an unmatched matrix pair leads to a less accurate reconstruction than with a matched transpose.

Acknowledgements. We thank the anonymous referees for their careful reading of the manuscript and for comments that helped to improve the presentation.

\section{REFERENCES}

[1] A. H. ANdersen AND A. C. KAK, Simultaneous algebraic reconstruction technique (SART): A superior implementation of the ART algorithm, Ultrasonic Imaging, 6 (1984), pp. 81-94.

[2] H. H. Bauschke, J. Y. Bello Ceuz, T. T. A. Nghia, H. M. Phan, and X. Wang, Optimal rates of linear convergence of relaxed alternating projections and generalized DouglasRachford methods for two subspaces, Numer. Algo., 73 (2016), pp. 33-76.

[3] Å. BJÖrck, Numerical Methods for Least Squares Problems, SIAM, Philadelphia, PA, 1996.

[4] Y. Censor, T. Elfving, G. T. Herman, and T. Nikazad, On diagonally relaxed orthogonal projection methods, SIAM J. Sci. Comput., 30 (2008), pp. 473-504.

[5] Y. Censor, D. Gordon, And R. Gordon, BICAV: an inherently parallel algorithm for sparse systems with pixel-dependent weighting, IEEE Trans. Med. Imag. 20 (2001), pp. 1050-1060.

[6] P. P. B. Eggermont, G. T. Herman, and A. Lent, Iterative algorithms for large partitioned linear systems, with applications to image reconstruction, Lin. Alg. Appl., 40 (1981), pp. $37-67$.

[7] T. Elfving, P. C. Hansen, and T. Nikazad, Semi-convergence properties of Kaczmarz's method Inverse Problems, 30 (2014), 055007.

[8] T. Elfving, P. C. HANSEn, And T. NikAzAd, Convergence analysis for column-action methods in image reconstruction, Numer. Algo., 74 (2017), pp. 905-924.

[9] T. Elfving and T. Nikazad, Properties of a class of block-iterative methods, Inverse Problems, 25 (2009), 115011.

[10] T. Elfving, T. NikAzad, And C. Popa, A class of iterative methods: semi-convergence, stopping rules, inconsistency, and constraining; in Biomedical Mathematics: Promising Directions in Imaging, Therapy Planning, and Inverse Problems, Y. Censor, M. Jiang, and G. Wang (Eds.), Medical Physics Publishing, Madison, WI, 2010.

[11] H. W. Engl, M. Hanke, and A. Neubauer, Regularization of Inverse problems, Kluwer, Dordrecht, 2000.

[12] L. Flores, V. VIDAL, AND G. Verdú, System matrix analysis for computed tomography imaging, PLoS ONE 10 (2015), e0143202. doi: 10.1371/journal.pone.0143202.

[13] A. Greenbaum, Iterative methods for solving linear systems, SIAM, Philadelphia, PA, 1997.

[14] P. C. HAnsen and M. Saxild-HAnsen, AIR Tools - A MATLAB package of algebraic iterative reconstruction methods, J. Comp. Appl. Math., 235 (2012), pp. 2167-2178.

[15] G. T. Herman, Fundamentals of computerized tomography, 2nd Ed., Springer, Dordrecht, 2009.

[16] P. Joseph, An improved algorithm for reprojecting rays through pixel images, IEEE Trans. Med. Imaging., 1 (1982), pp. 192196.

[17] A. C. Kak And M. Slaney, Principles of Computerized Tomographic Imaging, SIAM, Philadelphia, 2001.

[18] S. Kindermann And A. LeITÃo, Convergence rates for Kaczmarz-type regularization methods, Inverse Probl. Imaging, 8 (2014), pp. 149-172.

[19] F. NATTERer, The Mathematics of Computerized Tomography, SIAM, Philadelphia, PA, 2001.

[20] W. J. Palenstijn, K. J. Batenburg, and J. SiJbers, Performance improvements for iterative electron tomography reconstruction using graphics processing units (GPUs), J. Structural 
Biology, 176 (2011), pp. 250-253, 2011.

[21] Y. SAAD, Iterative Methods for Sparse Linear Systems, 2nd Ed., SIAM, Philadelphia, PA, 2003.

[22] X. SHI, Y. WeI, AND W. ZHANG, Convergence of general nonstationary iterative methods for solving singular linear equations, SIAM J. Matrix Anal. Appl., 32 (2011), pp. 72-89.

[23] R. Siddon, Fast calculation of exact radiological path length for a three dimentional CT array, Med. Phys., 12 (1985), pp. 252-255.

[24] H. H. B. Sørensen And P. C. HAnsen, Multicore performance of block algebraic iterative reconstruction methods, SIAM J. Sci. Comput., 36 (2014), pp. C524-C546.

[25] G. L. Zeng And G. T. GullberG, Unmatched projection/backprojection pairs in iterative reconstruction algorithms, IEEE Trans. Medical Imaging, 19 (2000), pp. 548-555.

[26] F. XU AND M. Mueller, A comparative study of popular interpolation and integration methods for use in computed tomography, in Proc. 3rd IEEE Int. Symp. on Biomed. Imag.; Nano to Macro, pp. 1252-1255, 2006.

[27] W. van Aarle, W. J. Palenstijn, J. Cant, E. Janssens, F. Bleichrodt, A. Dabravolski, J. De Beenhouwer, K. J. Batenburg, and J. Sijbers, Fast and flexible X-ray tomography using the ASTRA Toolbox, Optics Express, 24 (2016), pp. 25129-25147. 